J. Phys. IV France 127 (2005) 25-31

(C) EDP Sciences, Les Ulis

DOI: $10.1051 /$ jp4:2005127005

\title{
Quelle source pour la lithographie dans I'EUV ?
}

\author{
T. Ceccotti \\ Groupe d'Application des Plasmas, CEA-DSM/DRECAM/SPAM, CE de Saclay, \\ 91191 Gif-sur-Yvette, France
}

\begin{abstract}
Résumé. L'impressionnante évolution des performances des circuits intégrés (CI) ces trente dernières années, répond à la désormais célèbre loi de Moore. Selon la prédiction faite en 1975 par le co-fondateur d'Intel Gordon Moore et qui n'a jamais été contredite, le nombre de transistors dans un CI allait doubler tous les 18 mois. De simple intuition, la loi de Moore est devenue un impératif à respecter pour l'industrie des CI et des semi-conducteurs en général. La continuité dans les années à venir d'une telle progression technologique permettrait à ce secteur économique de garder, voire augmenter, toute son importance actuelle.

Augmenter le nombre des transistors dans les CI signifie principalement réduire leur taille caractéristique de gravure et par conséquent la longueur d'onde utilisée. Depuis 2000, la lithographie dans l'extrême ultraviolet (EUVL) à $13.5 \mathrm{~nm}$ est considérée comme la plus prometteuse parmi les technologies appelées à remplacer la lithographie actuelle qui utilise du rayonnement laser à $193 \mathrm{~nm}$ comme source de lumière.

La réalisation d'une machine lithographique industrielle utilisant du rayonnement EUV nécessite la résolution de nombreux problèmes technologiques qui font, depuis des années, l'objet de plusieurs programmes de recherche dans le monde. Une attention toute particulière est portée à la source de rayonnement EUV car ses caractéristiques, notamment de puissance et de propreté, vont décider du succès ou pas de l'EUVL.

Le cahier des charges d'une source EUV, les différents approches pour y répondre ainsi que leurs limites seront présentés ainsi qu'un état de l'art des performances des sources actuelles.
\end{abstract}

\section{INTRODUCTION}

L'utilisation de longueurs d'ondes de gravure de plus en plus courtes a permis jusqu'à aujourd'hui de réduire la dimension caractéristique $(\mathrm{CD})$ des traits gravés sur les wafers de silicium en augmentant ainsi le nombre de transistors par circuit intégré (CI) et, par conséquent, leurs performances. Vis-à-vis des enjeux économiques (le marché mondial des semi-conducteurs pourrait prochainement atteindre le chiffre record de 1000 milliards de dollars, environ le produit intérieur brut des USA), ne pas assurer la continuité de cette prouesse technologique connue sous le nom de Loi de Moore (doublement du nombre des transistors dans un CI tous les 18 mois) aurait des effets catastrophiques. La génération actuelle de machines lithographiques utilise le rayonnement à $193 \mathrm{~nm}$ produit par des lasers ArF pour réaliser des $C D$ de l'ordre de $90 \mathrm{~nm}$. La génération suivante d'excimers (laser $\mathrm{F}_{2}$ à $157 \mathrm{~nm}$ ) ne semble pas être capable d'assurer la relève a cause, entre autres, des problèmes intrinsèques de biréfringence et dilatation thermique des optiques $\mathrm{CaF}_{2}$ nécessaires pour travailler à cette longueur d'onde. Dans l'attente d'une nouvelle génération de lithographie (NGL) on espère pouvoir utiliser le rayonnement à $193 \mathrm{~nm}$ jusqu'à atteindre des CD de 45 voire $32 \mathrm{~nm}$ d'ici cinq ans environ. Cela pourrait se révéler possible en utilisant les mêmes techniques qui ont déjà permis d'obtenir des $\mathrm{CD}$ inférieures à la longueur d'onde utilisée (phase shift mask, optical proximity correction, off-axis illumination [1]) et avec l'introduction de la lithographie par immersion [2].

Depuis la moitié des années 80, les principaux acteurs du monde des semi-conducteurs ont commencé à se poser des questions sur l'avenir des actuelles techniques de gravure. Parmi différents candidats pour la NGL (lithographie par rayonnement X, par projection d'ions ou d'électrons, écriture directe par faisceau d'électrons), c'est finalement la lithographie dans l'extrême ultraviolet à $13.5 \mathrm{~nm}$ (EUVL) qui a été élue, en 2000, comme la plus prometteuse. L'EUVL peut être considérée pour certains aspects comme une évolution de la lithographie optique. Comme pour cette dernière, il s'agit de la reproduction 
d'un masque avec un facteur de réduction de 4, les méthodes d'amélioration de la résolution sont toujours applicables (hormis l'optique par immersion) et les équations qui donnent la résolution $(R)$ et la profondeur optique $(D O F)$ en fonction de la longueur d'onde $(\lambda)$ et de l'ouverture numérique $(N A)$ sont les mêmes ( $R=k_{1} \lambda / N A$ et $D o F=k_{2} \lambda /(N A)^{2}$ où $k_{1}$ et $k_{2}$ sont des paramètres propres au système optique). La mise en place de l'EUVL représente aussi une véritable révolution technologique à accomplir. Pour éviter la réabsorption du rayonnement, par exemple, il est impératif de travailler sous un très bon vide et employer un masque et des optiques multicouches en réflexion (et non plus en transmission). Aussi bien les optiques que le masque requièrent d'ailleurs une précision de réalisation jamais atteinte auparavant (moins de $10^{-3}$ defauts $/ \mathrm{cm}^{2}$ pour les masques et une rugosité inférieure à $0.1 \mathrm{~nm}$ pour les optiques). Cependant, en tête de la liste des obstacles qui restent à franchir, on trouve assurément la source de rayonnement. Depuis les premiers programmes de recherche consacrés à l'EUVL, il est apparu évident que le succès ou la faillite de l'EUVL comme remplaçant de l'actuelle lithographie optique reposent sur la réalisation d'une source aux caractéristiques bien précises. Des caractéristiques qui sont à la fois un impératif économique et un challenge technologique.

\section{SOURCES EUV : LA DEMANDE}

L'EUVL sera "bon marché" ou elle ne sera pas. Cela signifie que la rentabilité des futures machines EUVL doit être au moins comparable à celle des "steppers" utilisés actuellement. Cela peut se mesurer par le rapport entre le prix de la machine et la surface de silicium qu'elle est capable de graver par heure. Garder ce rapport constant, voire le réduire, permet d'offrir les produits dérivés de cette technologie à des prix favorables à une croissance de leur consommation et donc sustenter tous les secteurs économiques qui en dépendent. En effet, l'augmentation du prix avec le temps de machines lithographiques a été plus que compensée par leur capacité à assurer des flux de production de plus en plus importants [3] (30 wafers de $150 \mathrm{~mm}$ de diamètre par heure en 1990, 80 wafers de $200 \mathrm{~mm}$ de diamètre aujourd'hui). Pour les mêmes raisons, l'objectif pour les futures machines EUVL est, pour l'instant, fixé à 100-120 wafer de $300 \mathrm{~mm}$ de diamètre à l'heure.

Parmi les éléments qui constituent un appareil EUVL (Figure 1), c'est la source qui influence le plus son prix. On entend par prix tous les coûts qui sont associés à son achat et fonctionnement (alimentation, maintenance, personnel affecté etc... ). La source idéale se doit alors d'être puissante et à haute cadence (un haut débit de production signifie une réduction du coût de la surface unitaire de silicium gravé), propre (l'absence de débris réduits les coûts relatifs au remplacement des optiques avoisinantes et conséquente immobilisation de la machine) et avec une efficacité de conversion élevée. L'efficacité de conversion (CE) est définie comme le rapport entre l'énergie EUV collectable et celle utilisée par la source pour la produire. Augmenter le CE signifie baisser les frais d'alimentation de la source et en même temps réduire d'éventuels effets thermiques qui peuvent contribuer à la génération de débris.

Les constructeurs les plus importants de machines lithographiques (ASML, Canon et Nikon) mettent à jour environ tous les six mois un cahier des charges où sont spécifiées toutes les caractéristiques qu'une source doit posséder pour être "industriellement viable" [4]. Le dernier en date est reporté dans le Tableau 1. En voici les points principaux.

Longueur d'onde. Depuis la fin 2000, la longueur d'onde “officielle” pour l'EUVL a été fixée à $13.5 \mathrm{~nm}$. L'imposition d'une longueur d'onde bien précise à la communauté des développeurs de systèmes EUVL a été motivée, à la fois, par la disponibilité pour cette $\lambda$ d'optiques multicouches Mo-Si à haute réflectivité (environ $72 \%$ ), par l'intérêt suscité à l'époque par la raie $1 \mathrm{~s}-2 \mathrm{p} \mathrm{du} \mathrm{Li}^{2+}$ à $13.5 \mathrm{~nm}$ et finalement pour concentrer les efforts de tous dans une seule et unique direction de recherche.

Puissance. La puissance requise est de $115 \mathrm{~W}$ au foyer intermédiaire (tout de suite après le collecteur marqué par un 2 sur la Figure 1) dans une bande passante de $2 \%$ (valeur obtenue par convolution des courbes de réflectivité des nombreuses optiques multicouches rencontrées par les photons). 


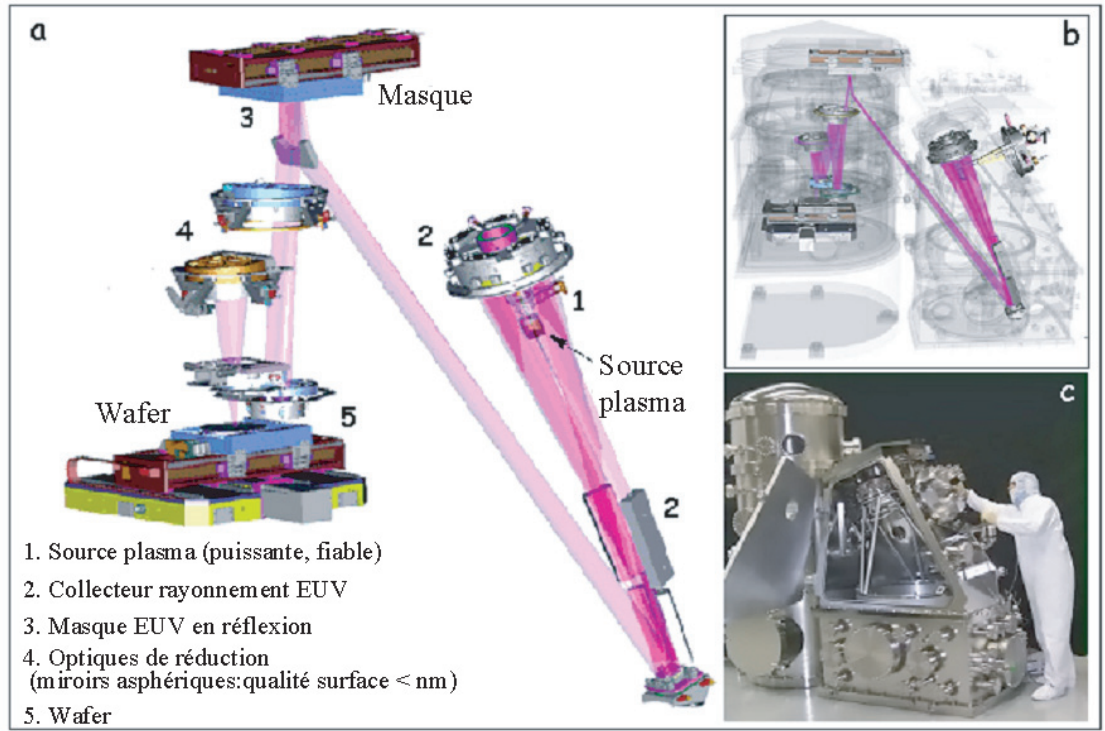

Figure 1. (a) Principaux éléments d'une machine EUVL (dans le cas spécifique, l'ETS au Sandia National Laboratory-USA), (b) leur implémentation à l'intérieur de la machine et (c) un aperçu de la réalisation finale. (Toutes les images proviennent du site web du SNL).

Tableau 1. Les indications concernant certaines propriétés de la source données par les producteurs de machines lithographiques lors du dernier EUV Source Workshop (février 2004) à Santa Clara (CA) [3].

\begin{tabular}{ll}
\hline Paramètre & Valeur requis \\
\hline Longueur d'onde & $13.5 \mathrm{~nm}$ \\
Puissance EUV (2\% bw) & $115 \mathrm{~W}$ \\
Cadence de tir & $7-10 \mathrm{KHz}$ \\
Stabilité en énergie (tir a tir) & $3 \%, 3 \sigma$ \\
Stabilité en énergie (intégrée) & $\pm 0.3 \%, 3 \sigma$ sur 50 impulsions \\
Stabilité spatiale & $<2 \%$ de la taille \\
Propreté & $\geq 30000$ heures* \\
Etendue & $1-3.3 \mathrm{~mm}^{2} \mathrm{sr}$ \\
\hline
\end{tabular}

*Au delà de ce temps, il est admis que l'action de la pollution de la source puisse provoquer une perte de $10 \%$ sur la réflectivité des optiques.

Cette puissance est imposée par les objectifs affichés de débit pour l'EUVL. Un débit de 100 wafers à l'heure correspond à 36 secondes par wafer, dont 9 secondes seulement sont consacrées à l'exposition, les autres étant nécessaires au positionnement du wafer.

Etant donnée la surface du wafer utilisable (environ 80\%) et la sensibilité de la résine photosensible $\left(5 \mathrm{~mJ} / \mathrm{cm}^{2}\right)$, on trouve que $2.9 \mathrm{~J} / \mathrm{cm}^{2}$ par wafer sont nécessaires, ce qui correspond à $0.32 \mathrm{~W}$. En parcourant à l'envers le chemin des photons et en tenant compte des pertes dues essentiellement à la réabsorption de l'EUV, à la réflectivité des miroirs et du masque et à l'efficacité du système optique entre le foyer intermédiaire et le masque, on trouve la valeur de $115 \mathrm{~W}$ (pour plus de détails, consulter par exemple [5]). Si l'on considère enfin l'efficacité et l'angle solide d'acceptance du collecteur, plus les pertes dues aux éventuels filtres spectraux et anti-débris et celles dues à la réabsorption, on découvre que la source doit produire entre 400 et $1000 \mathrm{~W}$ dans $2 \pi \mathrm{sr}$ et $2 \%$ de bande passante. 
Cadence et uniformité. Pour s'assurer de l'uniformité d'irradiation des wafers, on estime que chaque partie de leur surface doit recevoir au moins 50 "doses" de rayonnement, pourvu que la stabilité tir à tir soit de $3 \%$ sur $3 \sigma$ et que l'intégrale de l'énergie sur 50 impulsions présente une stabilité de $\pm 0.3 \%$ en $3 \sigma$. Dans un stepper standard, pour reproduire le motif sur le wafer, le système optique illumine une fente qui balaye à haute vitesse le masque. Si l'on prend des valeurs standards pour la vitesse de balayage $(200 \mathrm{~mm} / \mathrm{s})$ et pour la dimension de la fente $(1 \mathrm{~mm})$ et si l'on impose les 50 impulsions on trouve rapidement que la fréquence de répétition du rayonnement EUV doit être de $10 \mathrm{KHz}$.

Plusieurs programmes de recherche consacrés au développement d'une source qui puisse répondre à de tels critères ont vu le jour depuis la fin des années 90 [6]. Dès le départ, il a paru clair que seule une source plasma aurait pu sinon respecter au moins s'approcher du cahier des charges.

Deux philosophies de source se partagent les faveurs des développeurs : les plasmas produits par décharge (DPP) et les plasmas produits par laser (LPP) [7]. Pour les deux, il est aussi question du choix du matériau émetteur où il faut tenir compte aussi bien de ses propriétés émissives (qui influencent le $\mathrm{CE}$ et la puissance) que de ses qualités de propreté. Les deux types de sources, les matériaux émetteurs possibles et les solutions les plus significatives proposées sont présentés dans la section suivante.

\section{SOURCES EUV : L'OFFRE}

Les principaux modèles de source DPP (Figure 2) s'appuient tous sur le même principe [8]: un courant électrique de forte intensité circule entres deux électrodes à travers un cylindre rempli de gaz neutre. La décharge est accompagnée par un fort champ magnétique qui comprime le plasma ainsi formé dans la direction radiale. Les différents concepts se différencient essentiellement par leur géométrie et par les matériaux utilisés pour les électrodes et l'isolant. Ces différences affectent certains aspects technologiques tels que l'angle solide à disposition pour la collection du signal, la réabsorption du signal due au gaz qui peut être plus ou moins importante, la cadence de tir que l'on peut atteindre, la production de débris et la durée de vie des électrodes.

Les sources LPP se différencient par la nature de la cible et la structure du laser de pompe. Les jets sont privilégiés comme cible étant donnée la haute cadence de tir requise. En particulier, parmi les différents types de jet (Figure 3), c'est le micro-jet liquide qui semble aujourd'hui s'imposer comme le plus prometteur. Son utilisation permet d'avoir une bonne densité de matière au point d'impact du laser, d'éloigner ce dernier de l'injecteur en réduisant ainsi la production de débris et de réduire la présence de gaz, et donc la réabsorption d'EUV, dans l'enceinte. En ce qui concerne production du plasma on observe une tendance à préférer un système permettant le multiplexage, spatiale et/ou temporel, de plusieurs modules lasers de type POPA (power-oscillator-power-amplifier) à la place du plus classique mais aussi plus cher et moins versatile MOPA (master-oscillator-power-amplifier).

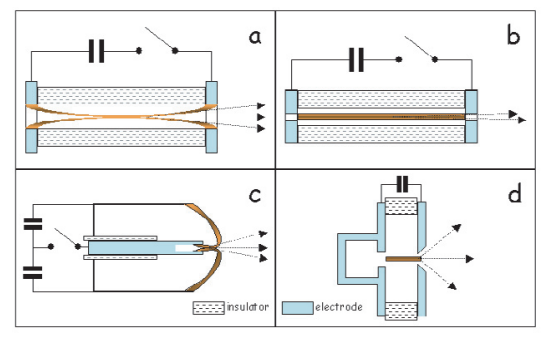

Figure 2. Les principales géométries de source DPP (a) le Z-pinch, (b) la décharge capillaire (c) le plasma focus et (d) la cathode creuse.

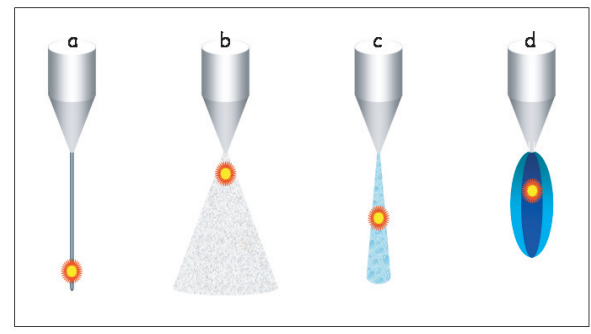

Figure 3. Principaux schémas de cibles jet : (a) le microjet liquide, (b) le jet d'agrégats, (c) le jet de gouttelettes et (d) le double jet concentrique. Le point montre la position de l'impact du laser : plus le jet est dense, plus le plasma est loin de l'injecteur, plus la charge thermique est faible. 


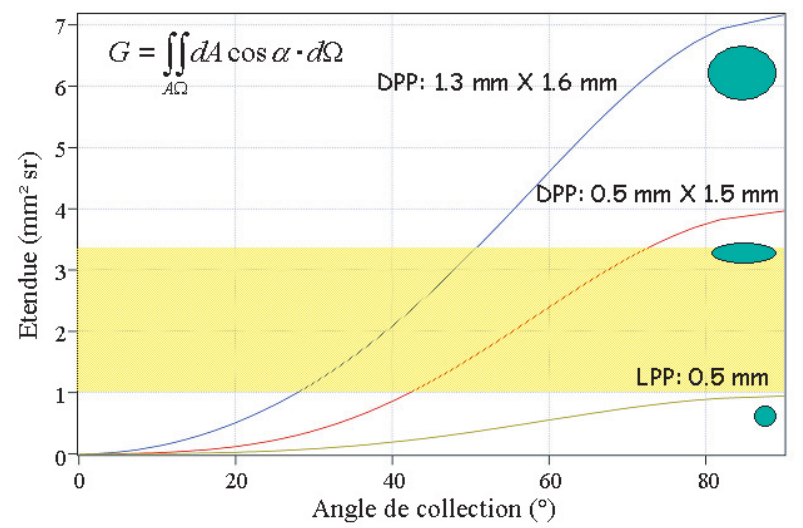

Figure 4. Calcul de l'étendue pour trois sources plasma différentes. La bande horizontale montre la fourchette imposée par le cahier des charges.

De façon générale, les DPP sont soumises à des problèmes de thermique plus importants, la distance moyenne entre le plasma et les matériaux autour étant 10 fois plus petite que pour les LPP. De la même façon, en plus du matériau émetteur, dans les DPP les électrodes et l'isolateur sont d'importantes sources de débris. Enfin, la taille moyenne d'un plasma dans une DPP $(\sim 0.5 \times 1.5 \mathrm{~mm})$ étant plus grande que dans une LPP $(\sim 0.5 \times 0.5 \mathrm{~mm})$, les limitations sur l'étendue prévue dans le cahier des charges empêchent de pouvoir multiplexer spatialement deux sources DPP ou plus, ce qui reste réalisable pour les LPP dans le cas ou la puissance d'une seule source serait insuffisante (Figure 4). De plus, les LPP offrent un bien meilleur angle solide de collection, environ 2 fois plus important que les DPP.

Pour résumer, les DPP présentent une relative simplicité associée à un faible coût (ce qui fait que des sources DPP consacrés à la métrologie sont actuellement déjà disponibles sur le marché) mais souffrent d'une plus élevée production de débris et de problèmes thermiques qui rendent difficile d'en augmenter la puissance EUV. Les LPP semblent pouvoir mieux atteindre l'objectif de puissance EUV mais ils affichent des coûts, notamment à cause de la partie laser, plus importants (environ un facteur 4) par rapport au DPP.

Aussi bien les DPP que les LPP souffrent d'un trop faible CE, ce qui, surtout pour les LPP et les lasers qu'ils nécessitent, se traduit par des coûts encore trop élevés. Des éléments comme l'oxygène et le lithium, jadis considérés comme des émetteurs potentiels d'EUV, ont été écartés à cause de leur CE insuffisant et/ou des problèmes de contamination des optiques. L'élément le plus utilisé dans tout type de source est aujourd'hui le xénon. Ses caractéristiques de gaz rare en font un candidat idéal pour une source propre et son spectre présente un ensemble de transitions 4p-4d et 4d-4f non résolues [9] (UTA [10]) centrées autour de $11 \mathrm{~nm}$. Cependant, son CE autour de 1\% laisse supposer qu'il pourra être difficilement utilisé dans le futur pour des machines industrielles. L'étain, grâce à ses caractéristiques émissives [11] (Figure 5), offre un CE au moins deux fois supérieur (l'UTA étant centré sur $13.5 \mathrm{~nm}$ ), mais à cause de sa nature métallique et de sa faible tension de vapeur, il pose de vrais problèmes vis à vis de la génération de débris et de la pollution des optiques. Il faut néanmoins garder à l'esprit que la marge de manœuvre pour réduire les coûts en espérant augmenter le CE est plutôt limitée. Si l'on considère comme limite infranchissable le cas idéal du corps noir et si l'on calcule le rapport entre l'énergie émise à $13.5 \mathrm{~nm}$ dans une bande passante de $2 \%$ et l'énergie à fournir on trouve une valeur maximale inférieure à $3 \%$ (Figure 6). Cela signifie qu' avec l'introduction éventuelle de l'étain on sera déjà assez proche du maximum que l'on peut espérer. C'est pour cette raison que les efforts pour rendre industrialisable (pas cher) l'EUVL se tournent vers d'autres directions. 


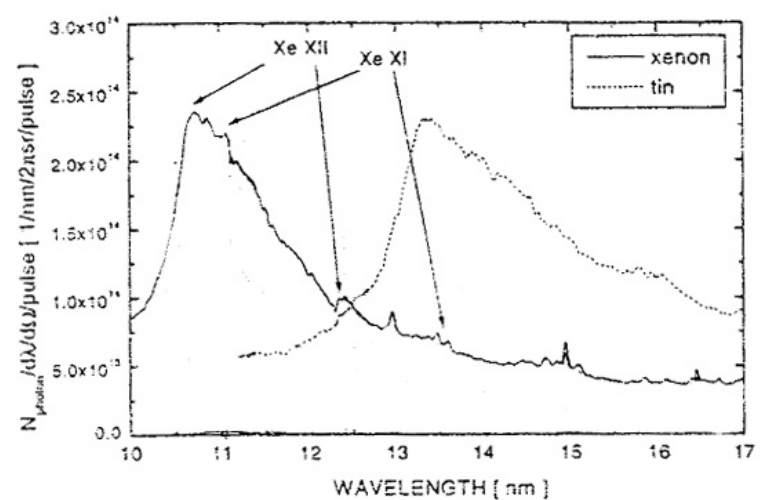

Figure 5. Spectres du xénon et de l'étain obtenus dans le cas de LPP par Schriever et al.

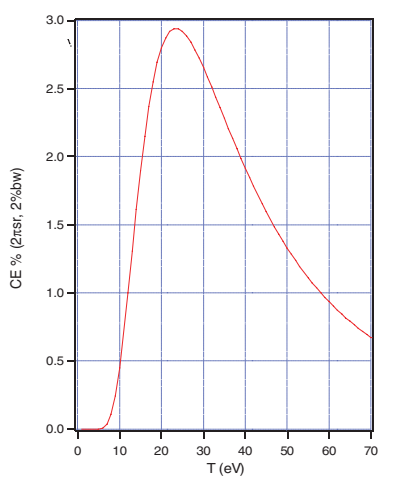

Figure 6. Efficacité de conversion en fonction de la température dans le cas d'un corps noir.

\section{CONCLUSIONS ET PERSPECTIVES}

Les craintes exprimées depuis le début des recherches autour de l'EUVL sur le rôle fondamental de la source dans la réussite de cette technologie se confirment. Le Tableau 2 recueille les caractéristiques des sources présentées lors du dernier EUV Source Workshop (février 2004) par les principaux développeurs. La comparaison avec le Tableau 1 montre que l'on est toujours loin des spécifications requises notamment en ce qui concerne la puissance EUV (environ 1 ordre de grandeur). Les limites physiques étant presque atteintes avec l'utilisation de l'étain, il reste des actions à mener dans d'autres directions pour essayer de répondre au cahier de charges. Il apparaitt clairement qu'il est souhaitable d'améliorer la collection de photons EUV (nouveau design, nouveaux matériaux) et de réduire les pertes dues aux filtres anti-débris et de pureté spectrale actuellement employés. De plus, une réduction du nombre des optiques et/ou une meilleure efficacité de ces dernières, l'augmentation du temps d'exposition et l'amélioration de la sensibilité des résines photosensibles sont autant d'éléments qui contribueraient à réduire la puissance EUV requise.

En ce qui concerne les DPP, le problème principale reste la réduction de la charge thermique qui limite dramatiquement la duré de vie des composants de la source tandis que pour les LPP il s'agit de pouvoir compter sur des lasers plus puissants, fiables et, surtout, moins chers (actuellement on estime à $100000 \$$ le prix de $1 \mathrm{~W}$ produit dans la bande passante [12]). Si les deux familles de sources restent en compétition, on peut vraisemblablement imaginer qu'on s'oriente néanmoins vers une génération de sources de puissance de type LPP, où la cible sera à base d'étain, beaucoup plus efficace que le xénon utilisé actuellement. Le principal obstacle qui se dresse à l'encontre des sources LPP (le prix des lasers de pompe) pourrait être éliminé par l'importante chute des prix des diodes laser de puissance prévue dans un futur proche [11].

Tableau 2. Le tableau affiche les plus récentes performances des principales sources actuellement en développement aux Etats Unis (Cymer et Plex), en Europe (Xtreme et Powerlase) et au Japon (EUVA). Le * indique une valeur seulement estimée et non pas mesurée.

\begin{tabular}{llllllll}
\hline & CYMER & EUVA & PLEX & XTREME & XTREME & POWERLASE & EUVA \\
\hline Source & DPP & DPP & DPP & DPP & LPP & LPP & LPP \\
Z & Xe & Xe & Xe & Xe & Xe & Xe & Xe \\
CE & 0.45 & 0.83 & 0.5 & 0.8 & 1.0 & 0.8 & 0.44 \\
EUV (IF) & $11.3 \mathrm{~W}$ & $2.7 \mathrm{~W} *$ & $7 \mathrm{~W}$ & $14.7 \mathrm{~W}$ & $1 \mathrm{~W}$ & $2.6 \mathrm{~W}$ & $0.9 \mathrm{~W}$ \\
Stabilité & $7 \%(1 \sigma)$ & $1.1 \%(1 \sigma)$ & $?$ & $<15 \%(3 \sigma)$ & $<15 \%(3 \sigma)$ & $<9 \%(3 \sigma)$ & $0.54 \%(1 \sigma)$ \\
(tir à tir) & & & & & & \\
Cadence & $2 \mathrm{KHz}$ & $2 \mathrm{KHz}$ & $2 \mathrm{KHz}$ & $4.5 \mathrm{KHz}$ & $5 \mathrm{KHz}$ & $3.5 \mathrm{KHz}$ & $10 \mathrm{KHz}$ \\
\hline
\end{tabular}


En 2000, on préconisait l'utilisation de l'EUVL comme outil de production industrielle pour des CD de $70 \mathrm{~nm}$ pour l'année 2005. Aujourd' hui, INTEL espère pouvoir utiliser l'EUVL pour des CD de $32 \mathrm{~nm}$ en 2009. Cela donne peut être un aperçu des difficultés qui se dressent encore sur le chemin vers l'utilisation de l'EUVL.

\section{Bibliographie}

[1] M.L. Rieger et al., Proceedings of the 38th conference on Design automation, p. 85 (2001)

[2] A. Hand, "Industry Takes a Hard Look at Immersion Lithography", Semiconductor International @ http: //www.e- insite.net/semiconductor, (2003).

[3] H.J. Lewinson, “Overview of lithography: challenges and metrologies”, @ http://www.eeel. nist.gov, (2003).

[4] Y. Watanabe, “Source requirements”, EUV Source Workshop 2004 @ http://www.sematech.org

[5] H. Franken, “Source requirements”, EUV Source Workshop 2003 @ http://www.sematech.org

[6] T. Ceccotti, "EUV lithography development in Europe: present status and perspectives", Proceedings of SPIE, Vol. 5196, pp. 57-70 (2004).

[7] M.A. Klosner and W.T. Silfvast, "High-temperature lithium metal-vapor capillary discharge extreme-ultraviolet source at 13.5 nm”, Appl. Optics 39 (21), p. 3678, 2000.

W.T. Silfvast and M.A. Klosner, "Intense xenon capillary discharge extreme-ultraviolet source in the 10-16-nm-wavelength region”, Opt. Lett. 23, p. 1609, 1998.

W.T. Silfvast et al., "High-power plasma discharge source at $13.5 \mathrm{~nm}$ and $11.4 \mathrm{~nm}$ for EUV Lithography”, Proc. SPIE 3676, p. 272, 1999.

M. McGeoch, "High-power extreme ultraviolet source based on a Z-pinch”, Proc. SPIE Vol. 3676, p. $697,1999$.

W.N. Partlo et al, "Development of an EUV (13.5 nm) light source employing a dense plasma focus in lithium vapor”, Proc. SPIE Vol. 3997, p. 136, 2000.

$\mathrm{K}$. Bergmann et al, "Highly repetitive, extreme-ultraviolet radiation source based on a gasdischarge plasma”, Appl. Opt. 38, p. 5413, 1999.

G. Schriever et al, "Extreme ultraviolet light generation based on laser-produced plasmas (LPP) and gas-discharge-based pinch plasmas: a comparison of different concepts”, Proc. SPIE Vol. 3997, p. 162, 2000.

$\mathrm{K}$. Bergmann et al, "Pinch-plasma radiation source for extreme-ultraviolet lithography with a kilohertz repetition frequency”, Appl. Opt. 39, p. 3833, 2000.

N.R. Fornaciari et al, "Development of an electric capillary discharge source", Proc. SPIE Vol. 3997, p. 120, 2000.

G.D. Kubiak et al, "Scale-up of a cluster jet laser plasma source for extreme ultraviolet lithography”, Proc. SPIE Vol. 3676, p. 669, 1999.

G.D. Kubiak et al, "High-power source and illumination system for extreme ultraviolet lithography”, Proc. SPIE Vol. 3767, p. 136, 1999.

[8] W. Neff et al, "Pinch Plasma Radiation Sources for the Extreme Ultraviolet", Contrib. Plasma Phys. 41, p. 589, 2001 and references therein.

[9] F. Gilleron et al., "Emissive properties of xenon ions from a laser-produced plasma in the 100-140 A spectral range: atomic-physics analysis of the experimental data”, J. Appl. Phys.

[10] J. Bauche et Bauche-Arnoult, Phys. Script., T40, p. 58 (1992).

[11] G. Schriever et al, J. Vac. Sci. Technol. B, 17, p. 2058 (1999).

M. Richardson et al, "The case for tin as en EUV source”, $2^{\text {nd }}$ Int. Symp. EUVL 2003 @ http://www.sematech.org

[12] M. Hatcher, “EUV sources shape up for competition”, Opto \& Laser Europe, 110, p. 22 (2003). 